\title{
Incidence of Morphea following Adjuvant Irradiation of the Breast in 2,268 Patients
}

\author{
Richard Partl ${ }^{\mathrm{a}}$ Peter Regitnig ${ }^{\mathrm{b}} \quad$ Katarzyna Lukasiak $^{\mathrm{a}} \quad$ Peter Winkler $^{\mathrm{a}}$ \\ Karin Sigrid Kapp ${ }^{a}$ \\ aDepartment of Therapeutic Radiology and Oncology, Medical University of Graz, Comprehensive Cancer Center \\ Graz (CCC), Graz, Austria; ${ }^{b}$ Institute of Pathology, Medical University of Graz, Comprehensive Cancer Center Graz \\ (CCC), Graz, Austria
}

\section{Keywords}

Morphea $\cdot$ Incidence $\cdot$ Radiotherapy $\cdot$ Irradiation $\cdot$ Breast

\begin{abstract}
Background: Morphea of the breast is an autoimmune reaction of the subcutaneous connective tissue which can be triggered by exposure to ionizing radiation. The literature suggests incidence rates of 1:500 to 1:3,000 which, however, do not seem to match the very small number of cases reported. Objectives: The aim of the present study was to determine the incidence of morphea following irradiation of the breast in order to generate more evidence about the frequency of this serious and mutilating complication. Method: Retrospective analysis of patient data who underwent adjuvant radiotherapy in the period 2009-2018 following breastconserving surgery and who made use of the recommended radiooncology follow-up examinations in 2018. Analysis was done by descriptive statistics. Results: Of a total of 5,129 patients who had undergone radiotherapy over a 10-year period, follow-up data were available in 2,268 patients. In 2,236 patients $(98.6 \%)$ the breast had been irradiated using conventional fractionation schemes with a total dose of 50-50.4 Gy; 32 (1.4\%) were given a total dose of 40.05 Gy in 15 fractions. During the observation period, 6 patients were diagnosed with morphea ( 4 unilateral and 2 bilateral) by punch
\end{abstract}

biopsies resulting in a cumulative incidence proportion of $0.26 \%$ (95\% Cl: $0.24-0.28)$, translating into 1 case for every 378 irradiated patients. Conclusions: In the case studies reported to date, morphea is described as a very rare complication. In contrast, our data suggest a cumulative incidence of 1:378, which is higher than other authors have estimated. This leads us to suspect that in a large number of patients, morphea is incorrectly diagnosed as an infection (in the early stages) and radiation-induced fibrosis (in later stages).

(c) 2019 S. Karger AG, Basel

\section{Introduction}

Postirradiation morphea (PIM) (synonyms: radiation-induced morphea, radiation-induced scleroderma, radiation port morphea, radiation port scleroderma, localized scleroderma, and circumscribed scleroderma) is described by most authors as a rare complication following radiotherapy $[1,2]$. Usually, it presents as an abruptonset circumscribed inflammation of the skin and underlying tissue, which progresses to a chronic stage with fibrotic transformation of the affected tissues. The connection between ionizing radiation and the occurrence of morphea was already noticed shortly after the discovery of X-rays by Konrad Roentgen; the condition was 
Table 1. Reports of localized morphea after adjuvant irradiation of the breast since 2009

\begin{tabular}{|c|c|c|c|}
\hline Author & $\begin{array}{l}\text { Cases, } \\
n\end{array}$ & $\begin{array}{l}\text { Time interval be- } \\
\text { tween radio-thera- } \\
\text { py and onset }\end{array}$ & Prior systemic treatment \\
\hline Herrmann et al. [1], 2009 & 1 & 1.5 years & Antihormonal therapy \\
\hline Morganroth et al. [4], 2013 & 1 & 6 years & Doxorubicin, cyclophosphamide, paclitaxel \\
\hline Laetsch et al. [5], 2011 & 3 & $<1$ year & $\begin{array}{l}1 \text { case: doxorubicin and cyclophosphamide, tamoxifen; } \\
2 \text { cases: no systemic treatment }\end{array}$ \\
\hline Wernicke et al. [6], 2011 & 1 & 1.5 years & Tamoxifen \\
\hline Alhathlool et al. [7], 2012 & 1 & 2.7 years & Anastrozole \\
\hline Lim et al. [8], 2014 & 1 & 7 months & Epirubicin, cyclophosphamide, docetaxel \\
\hline García-Arpa et al. [9], 2015 & 1 & 1 year & Chemotherapy (not specified), letrozole \\
\hline Yanaba et al. [10], 2015 & 1 & 3 months & Not recorded \\
\hline Dyer et al. [2], 2016 & 2 & 3-4 months & 1 case: chemotherapy (not specified) \\
\hline Chu et al., [11] 2017 & 1 & 10 months & Not recorded \\
\hline Gonzalez-Ericsson et al. [12], 2018 & 1 & 1.3 years & Cisplatin, paclitaxel \\
\hline Friedman et al. [13], 2018 & 3 & $4,5,7$ years & $\begin{array}{l}\text { Case 1: neoadjuvant chemotherapy (not specified), adjuvant } \\
\text { tamoxifen; case 2: adjuvant tamoxifen; case } 3 \text { : none }\end{array}$ \\
\hline Peterson et al. [14], 2018 & 1 & 5 months & Not recorded \\
\hline Papanikolaou et al. [15], 2018 & 1 & 4 months & Not recorded \\
\hline Partl et al. [16], 2018 & 1 & 3 months & Anastrozole, CDK4/6 inhibitor (Abemaciclib) \\
\hline Machan et al. [17], 2019 & 1 & 4 years & 5-fluorouracil, cyclophosphamide, docetaxel, tamoxifen \\
\hline
\end{tabular}

first described by Crocker [3] in 1905. Notably, the large majority of cases arise following irradiation of the mammary gland. In the last 10 years, only a total of 21 patients with morphea following breast irradiation have been reported in the literature [1, 2, 4-17] (Table 1). The pathological mechanism leading to PIM is not yet understood; it is suspected that it originates in a dysregulation of an immune reaction in genetically susceptible individuals. A review of the hypotheses on the pathology was recently published [16]. To our knowledge only three reports on the frequency of PIM are available, which estimate the prevalence of irradiated breast cancer patients at $1: 500$ to $1: 3,000[13,18,19]$, though this seems to conflict with the rare observations of the condition in clinical practice $[2,3]$. If these prevalence estimates are accurate, it would suggest that this complication is identified incorrectly in a substantial proportion of cases - thus depriving these patients of therapy that could stop or at least slow the progression of this painful and mutilating complication. The aim of the present work was to analyze the incidence of morphea in patients following adjuvant radiotherapy of the breast in our patient cohort.

\section{Materials and Methods}

We recommend to all patients who receive radiotherapy at our clinic to make use of regular radiooncological follow-up examinations for at least 10 years after radiotherapy. The follow-up examinations are done to monitor recurrence and to assess possible radiation-induced side effects as early as possible. If there is a suspicion of morphea, and to exclude other differential diagnoses (e.g., an inflammatory recurrence of cancer, an infection), a punch biopsy is performed. The aim of the present retrospective observational study was to review the data on the patients with carcinoma of the breast who underwent adjuvant radiotherapy at our clinic in the period 2009-2018 and who came for follow-up examinations, in order to calculate the cumulative incidence proportion (IP) of morphea. For study purposes the following data were extracted from the electronic patient records: age at the time of radiotherapy, tumor histology, grading, receptor status (estrogen, progesterone), TNM staging, concomitant systemic treatment, fractionation and radiation dose, length of time between radiotherapy and presentation with morphea, length of time between radiotherapy and definitive histological diagnosis, presence of any autoimmune diseases, type of topical or systemic treatment of the morphea, and follow-up time.

In all patients, the irradiation of the affected breasts was carried out using a 3D conformal technique with $6 \mathrm{MeV}$ photons. Depending on the breast volume, the total dose was 50.0-50.4 Gy and was applied in 25-28 fractions with or without a boost to the tumor bed or 40.05 Gy in 15 fractions [20], delivered 5 days per week. Radiation-induced acute skin toxicity was classified according to the Common Terminology Criteria for Adverse Events (CTCAE) version 4.03 [21].

Analysis was done by descriptive statistics. IP was defined as the number of morphea cases divided by the total number of patients $(N)$ seen for follow up. The IP was then multiplied by 100 to gain the IP percentage (IP\%). 95\% CI for the IP\% was calculated using the following formula:

$$
95 \% C I=I P \% \pm 1.96 \times \sqrt{\frac{I P \% \times(1-I P \%)}{N}} .
$$

For numerical data, medians and ranges were calculated. Categorical data are reported as absolute and relative frequencies. Statistical analysis was done using IBM SPSS Statistics (Release 23.0.0 2015; Chicago, IL, USA). 
Table 2. Patient- and tumor-related parameters of 2,268 patients with radiooncological follow-up data

\begin{tabular}{lc}
\hline Parameter & $n(\%)$ \\
\hline Median age (range), years & $60.2(23.1-92.2)$ \\
Histology & \\
$\quad$ Ductal carcinoma in situ & $146(6.4)$ \\
Invasive carcinoma (no special type) & $1,888(83.3)$ \\
Invasive lobular carcinoma & $166(7.3)$ \\
Mucinous carcinoma & $40(1.8)$ \\
Others & $28(1.2)$ \\
Grading & \\
G 1 & $312(13.8)$ \\
G 2 & $1,213(53.5)$ \\
G 3 & $743(32.7)$ \\
T classification & \\
pTis & $146(6.4)$ \\
pT 1 & $1,707(75.3)$ \\
pT 2 & $351(15.5)$ \\
pT3 & $57(2.5)$ \\
pT4 & $7(0.3)$ \\
N classification & \\
pN 0 & $2,043(90.1)$ \\
pN 1 & $190(8.4)$ \\
pN 2-3 & $35(1.5)$ \\
RTx dose & $32(1.4)$ \\
40.05 Gy & $2,236(98.6)$ \\
$\geq 50$ Gy & $2,090(92.2)$ \\
Tumor boost & \\
\hline
\end{tabular}

$\mathrm{RTx}$, radiotherapy.

\section{Results}

In the period 2009-2018, a total of 5,129 patients with invasive or preinvasive carcinoma of the breast underwent adjuvant radiotherapy at our clinic. In the observation period of January-December 2018, a total of 2,268 $(44.2 \%)$ patients were seen by us for their recommended follow-up examination and were included in our analysis. These patients had a median age at the time of radiotherapy of 60.2 years (range: 23.1-92.2). In 2,236 (98.6\%) of the patients, the breast had been irradiated using conventional fractionation with a total dose of 50-50.4 Gy; 2,090 of these patients (93.5\%) also received a boost to the tumor bed ranging from 10 to $14 \mathrm{~Gy}$. The remaining $32 \mathrm{pa}-$ tients $(1.4 \%)$ had hypofractionated radiotherapy with a total dose of $40.05 \mathrm{~Gy}$. The median time of follow-up was 3.7 years (range: 0.7-10). Patient characteristics are shown in Table 2 . In 6 patients the biopsy revealed a diagnosis of morphea (Table 3). In 2 of the patients, the morphea was bilateral after irradiation of both breasts. The cumulative incidence of morphea was 1 case for every 378 irradiations of the breast with a morphea IP\% of $0.26 \%$ (95\% CI: $0.24-0.28$ ). The interval between the end of radiotherapy and the clinical identification of a possi-

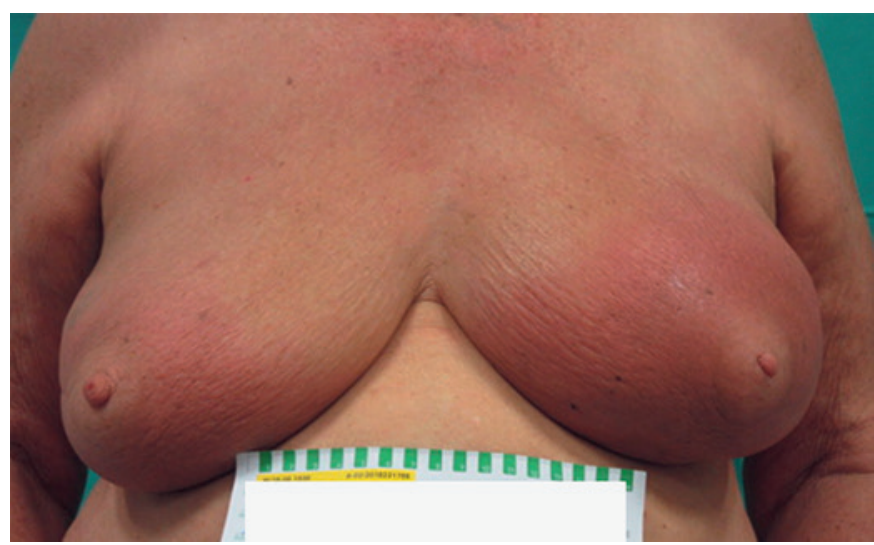

Fig. 1. Four months after bilateral breast irradiation an abrupt onset of inflammation occurred. Both breasts were swollen, slightly indurated, and erythematous patches could be observed. The clinical suspicion of inflammatory morphea was confirmed by punch biopsy.

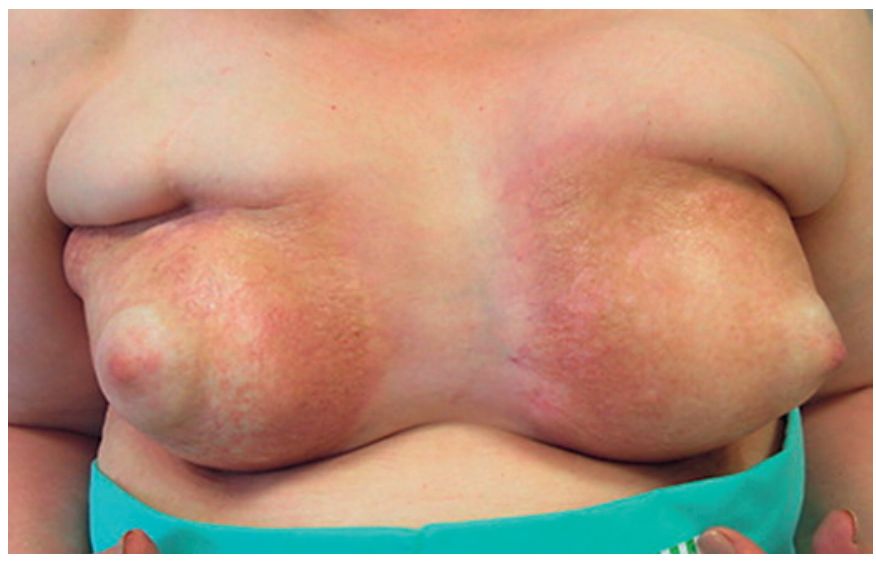

Fig. 2. Clinical picture of a bilateral morphea in the sclerotic phase 35 months after irradiation. Both breasts were reduced in size and hardened with significant hypo- and hyperpigmentation and skin telangiectasia. The nipples were typically unaffected by the reaction.

ble morphea was between 3 and 10 months. The breast volume of these 6 patients at the time of radiotherapy was between 1,022 and $2,920 \mathrm{ccm}$. The diagnosis of morphea was made in different phases of the disease. To illustrate these, we will describe the cases of one patient with morphea in the inflammatory phase and one patient with morphea in the sclerotic phase.

Patient 2 (inflammatory phase): a 79-year-old patient with bilateral carcinoma of the breast (right: $\mathrm{pT} 1 \mathrm{pN} 1 \mathrm{M} 0$, no specific type [NST], G3, ER and PR pos., Her2/neu neg.; left: pT1 pN0 M0, NST, G1, ER and PR pos., Her2/ neu neg.) underwent breast-conserving surgery in 2018, and was given adjuvant radiotherapy of 40.05 Gy in 15 fractions, while taking anastrozole concomitantly. Four months after complete healing of the acute radiodermatitis (CTCAE grade 1), the patient experienced an abrupt 


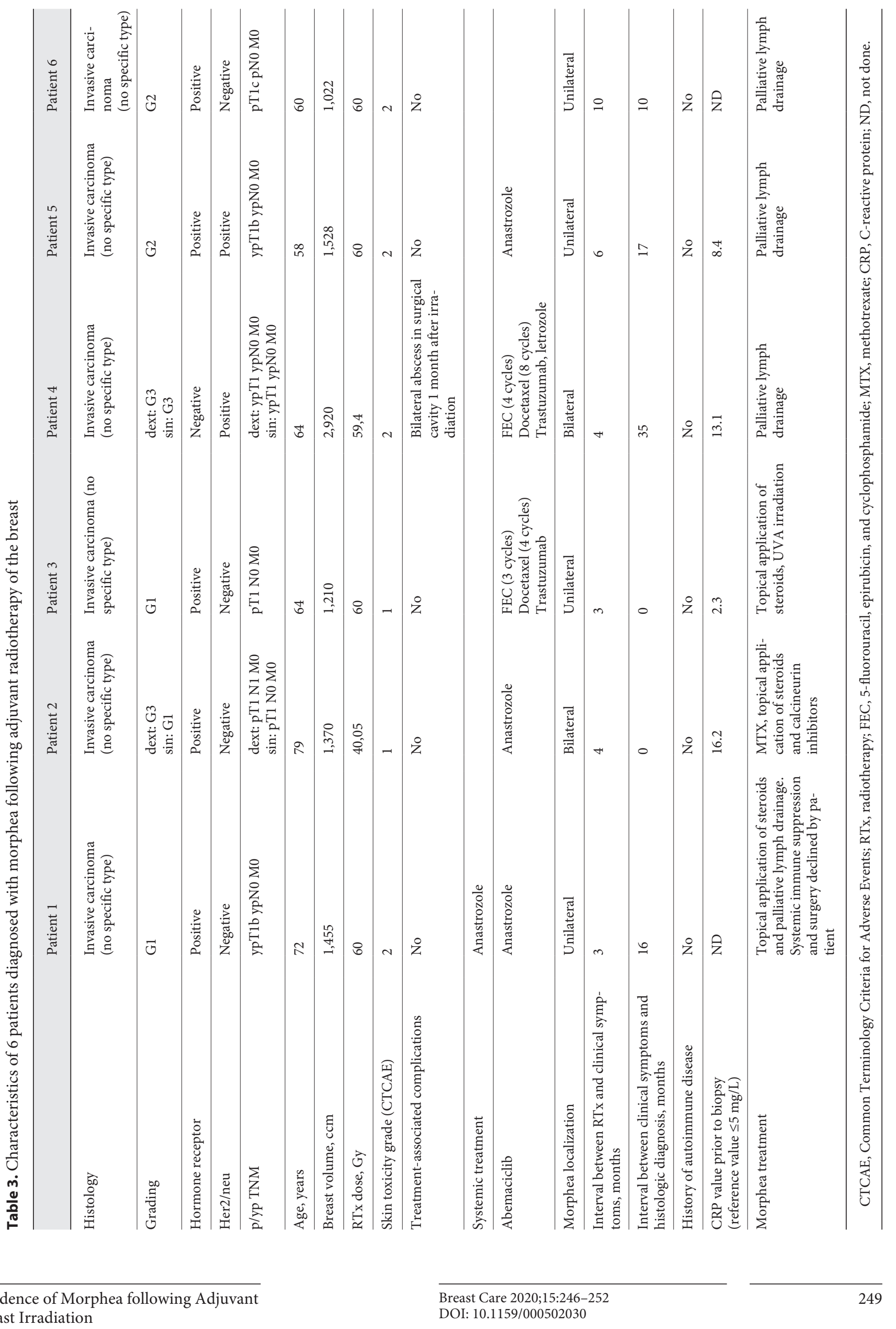


onset of a bilateral inflammatory reaction (Fig. 1), which was examined by biopsy within 10 days. The punch biopsy confirmed histomorphological changes in accordance with morphea. We prescribed systemic treatment with methotrexate and topical treatment with cortisone and calcineurin inhibitors, which achieved a reduction of the inflammatory reaction within 4 weeks. The treatment is currently ongoing and is well tolerated by the patient.

Patient 4 (sclerotic phase): a 64-year-old patient was diagnosed with bilateral carcinoma of the breast (NST, G3, Her2/neu pos.) in 2014. Following neoadjuvant chemotherapy with three cycles of 5-fluorouracil, epirubicin, and cyclophosphamide (FEC) and four cycles of docetaxel, the patient underwent bilateral lumpectomy and axillary dissection, revealing a ypT1a ypN0 stage on the right and a yp T1c ypN0 on the left. This was followed by bilateral conventionally fractionated radiotherapy, which was well tolerated apart from an acute skin reaction classified as CTCAE grade 2. Trastuzumab was begun as a further adjuvant therapy. One month after ending the radiotherapy, abscesses with Staphylococcus aureus appeared in the surgical cavity on the right and in the lymphadenectomy scar on the left; these were treated surgically. Four months after radiotherapy, the left breast appeared substantially enlarged and hardened. The right breast was unremarkable at this point of time. At a follow-up examination 15 months after radiotherapy, both breasts were massively hardened, with reddening and hyperpigmentation of the skin. Due to the pronounced lymphedema, regular drainages had been performed up to that point. The clinical suspicion of morphea arose in the "burn-out phase" of both breasts (Fig. 2) and was confirmed by punch biopsy in July 2018, 35 months after onset of the first symptoms. In view of the advanced state of the disease, anti-inflammatory therapy no longer appeared very promising. Symptomatic treatment by means of regular lymph drainage continues. The patient declined a surgical intervention which had been suggested by her breast surgeon.

\section{Discussion/Conclusion}

Worldwide, 1.67 million patients are diagnosed with new cases of breast cancer each year [22]. This high incidence is a global health problem and is a significant cause of mortality [23]. Best practice therapy for breast cancer takes account of tumor stage, tumor size, steroid receptors, Her-2/neu expression, mitotic index, and patient preferences and allows breast-conserving approaches to be used in a high proportion of cases [24, 25]. Adjuvant irradiation of the breast is an integral part of the treatment concept following breast-conserving surgeries and is one of the most frequent indications of all adjuvant radiotherapy. PIM is described by most authors as a rare side effect. The symptoms appear abruptly after an asymptomatic interval of at least a month to several years. In the initial inflammatory phase, edematous and erythematous plaques form in the skin. In the subsequent sclerotic phase, the breast tissue contracts and forms scar tissue and causes severe pain and cosmetic problems. The changes are usually confined to the irradiated zone, but in rare cases they can extend beyond it and may even become generalized [26-28]. Typically, the nipple is not affected by the reaction, which may serve as a lead indication for the presence of morphea (Fig. 1,2). In the inflammatory phase, the differential diagnoses inflammatory tumor recurrence, infection, and radiation recall dermatitis should be excluded. In the sclerotic phase, chronic radiodermatitis and radiation-induced fibrosis should be considered. According to Spalek et al. [27] histological confirmation is crucial in distinguishing clinical similarlooking pathologies. In the sclerotic stage, the diagnosis can be challenging because distinct histomorphological criteria are missing and the underlaying cause of fibrotic transformation is frequently not clearly visible. Therefore, in unclear cases it is important to make the histological diagnosis in combination with the clinical picture. In 3 of our patients the final diagnosis could only be made in conjunction with the distinct clinical picture.

Until now, only three authors have investigated the frequency of morphea following breast irradiation. Davis et al. [19] described 6 cases of PIM from 3,000 patients who had received adjuvant breast irradiation. This estimate of the incidence was supported by Bleasel et al. [18], who found 4 cases among 1,500 irradiated patients. These data show that PIM is not as rare as is usually described in the literature. The data we have collected support the estimated incidence of at least 1:378 (IP 0.26\%) breast cancer patients irradiated. The findings by Davis et al. [19] (IP 0.2\%) and Bleasel et al. [18] (IP 0.26\%), as well as our findings, contradict a recent analysis by Friedman et al. [13] in which only 3 cases of morphea were identified following 12,000 cases of breast irradiation in the period 2005-2016, which translates into an IP of $0.025 \%$. The fact that ion irradiation can trigger morphea at any site is supported by the incidence of idiopathic morphea in the nonirradiated population, which is considered lower at 2.7:100,000 persons per year [29], and translates into an IP of $0.0027 \%$. The authors believe that the true number of patients developing morphea after irradiation of the breast is incorrectly diagnosed in a substantial number of cases. We are aware of the weaknesses of our study which is based on only $44 \%$ of our patients who made use of radiooncological follow-up examinations. We cannot account for the possible rate of morphea in patients who chose to have their examinations elsewhere or declined follow-up examinations for reasons unknown. Neither can we rule out our own shortcomings in misdiagnosis in 
cases of minor symptoms or in the state of fibrotic transformation.

Until now it has not been possible to identify any risk factors that could influence the development of morphea. In particular, it was not possible to find any relationship between the irradiation parameters, the acute reactions to irradiation, age, or neoadjuvant or concomitant systemic therapy $[18,30]$. However, there could be a link between autoimmune diseases and an elevated risk of morphea [17]. According to Diago et al. [31], 4 out of 6 patients had rheumatoid arthritis, Sjögren syndrome, vitiligo, or Crohn's disease. In our 6 patients no history of autoimmune comorbidities was observed. There was a lack of other clinical local or general symptoms or abnormalities in laboratory tests prior to radiotherapy. What was noticeable was that our affected patients did have an aboveaverage breast volume (range: 1,022-2,920 ccm). The size of the subcutaneous volume affected by the radiation might be a contributing factor to the emergence of morphea. In 3 out of 6 patients a slight increase of CRP at the time of biopsy was observed (Table 3 ).

In most cases, the onset of PIM was within 1 year after finishing radiotherapy, but intervals of 1 month to 32 years have been reported [32]. Remarkably, the large majority of cases are related to irradiation of carcinomas of the breast. A possible explanation for this could be the unavoidable inclusion of skin and subcutaneous tissue in the irradiated volume and also the fact that the radiation target is easy to examine visually [33].

The success of treatment is related to the stage of morphea. In order to prevent irreversible tissue damage, a therapy should be initiated as soon as possible after histological confirmation of the diagnosis. In the acute inflammatory stage, topically and systemically applied immune suppressants are recommended. For symptomatic alleviation of fibrosis in the late stage local application of heparin, hyaluronidase, ultraviolet A irradiation, or pen- icillin have been recommended. The various therapeutic approaches have been evaluated in the largest PIM cohort worldwide [34]. Systemic treatment with methotrexate or ultraviolet B phototherapy achieved the best responses.

Our study suggests that the incidence of morphea is much higher than previously reported. Practitioners in oncology are advised to consider this diagnosis in its early stage and to confirm it by biopsy since histopathological diagnosis might be difficult in its sclerotic stage. It is an essential condition that the biopsy includes the underlying tissue of the skin and it might be helpful to provide the pathologist with information on prior irradiation and systemic therapy, onset of symptoms, and clinical picture.

\section{Statement of Ethics}

The subjects gave their written informed consent. The study protocol was approved by the local research institute's committee on human research.

\section{Disclosure Statement}

The authors have no conflicts of interest to declare.

\section{Funding Sources}

The authors did not receive any funding for this study.

\section{Author Contributions}

Richard Partl: idea, data acquisition, data analysis, and manuscript writing.

Peter Regitnig: histopathological examinations, review, and critical feedback.

Katarzyna Lukasiak, Peter Winkler, and Karin Sigrid Kapp: data acquisition, review, and critical feedback.

\section{References}

1 Herrmann T, Günther C, Csere P. Localized morphea-a rare but significant secondary complication following breast cancer radiotherapy. Case report and review of the literature on radiation reaction among patients with scleroderma/morphea. Strahlenther Onkol. 2009 Sep;185(9):603-

2 Dyer BA, Hodges MG, Mayadev JS. Radiation-induced morphea: an underrecognized complication of breast irradiation. Clin Breast Cancer. 2016 Aug;16(4):e141-3.

3 Crocker HR. Diseases of the skin. Philadelphia. Blakistons; 1905. p. 633.

4 Morganroth PA, Dehoratius D, Curry H, Elenitsas R. Postirradiation morphea: a case report with a review of the literature and summary of clinicopathologic differential diagno- sis. AmJDermatopathol. AmJDermatopathol. 2013 [Epub ahead of print].

5 Laetsch B, Hofer T, Lombriser N, Lautenschlager S. Irradiation-induced morphea: $\mathrm{X}$ rays as triggers of autoimmunity. Dermatology. 2011;223(1):9-12.

6 Wernicke AG, Goltser Y, Trichter S, Sabbas A, Gaan J, Swistel AJ, et al. Morphea as a consequence of accelerated partial breast irradiation. Clin Breast Cancer. 2011 Mar;11(1):67-70.

7 Alhathlool A, Hein R, Andres C, Ring J, Eberlein B. Post-Irradiation Morphea: case report and review of the literature. J Dermatol Case Rep. 2012 Sep;6(3):73-7.

8 Lim D, Johnston S, Novakovic L, Fearfield L. Radiation-induced morphoea treated with UVA-1 phototherapy. Clin Exp Dermatol. 2014 Jul;39(5):612-5.
9 García-Arpa M, Lozano-Martín E, Rodríguez CR, Rodríguez-Vázquez M. Morphea following radiation therapy in a patient with breast cancer. Actas Dermosifiliogr. 2015 Apr; 106(3):243-5.

10 Yanaba K, Umezawa Y, Nakagawa H. A case of radiation-induced generalized morphea with prominent mucin deposition and tenderness. Am J Case Rep. 2015 May;16:279-82.

$11 \mathrm{Chu} \mathrm{CH}$, Cheng YP, Liang CW, Chiu HC, Jee $\mathrm{SH}$, Lisa Chan JY, et al. Radiation recall dermatitis induced by topical tacrolimus for post-irradiation morphea. J Eur Acad Dermatol Venereol. 2017 Feb;31(2):e80-1.

12 Gonzalez-Ericsson PI, Estrada MV, Al-Rohil R, Sanders ME. Post-irradiation morphoea of the breast: a case report and review of the literature. Histopathology. 2018 Jan;72(2):342-50. 
13 Friedman O, Barnea Y, Hafner A. Underdiagnosed and disfiguring - Radiation-induced morphea following breast cancer treatment. Breast. 2018 Jun;39:97-100.

14 Petersen E, Yazdani L, Hymes SR. A case of radiation-induced bullous morphea/lichen sclerosus overlap in a breast cancer patient. Rep Pract Oncol Radiother. 2018 Jan-Feb; 23(1):47-9.

15 Papanikolaou M, Tsianou Z, Skellett AM, Murphy J, Millington GW. Radiotherapy-induced morphoea of the breast responding to photodynamic therapy. Clin Exp Dermatol. 2018 Jun;43(4):506-8.

16 Partl R, Regitnig P, Tauber G, Pötscher M, Bjelic-Radisic V, Kapp KS. Radiation-induced morphea - a rare but severe late effect of adjuvant breast irradiation: case report and review of the literature. Strahlenther Onkol. 2018 Nov; 194(11):1060-5.

17 Machan A, Oumakhir S, Khalidi M, Hjira N, Boui M. Radiation-induced morphea: autoimmunity as a risk factor. Neth J Med. 2019 Jan;77(1):29-31.

18 Bleasel NR, Stapleton KM, Commens C, Ahern VA. Radiation-induced localized scleroderma in breast cancer patients. Australas J Dermatol. 1999 May;40(2):99-102.

19 Davis DA, Cohen PR, McNeese MD, Duvic M. Localized scleroderma in breast cancer patients treated with supervoltage external beam radiation: radiation port scleroderma. J Am Acad Dermatol. 1996 Dec;35(6):923-7.
20 Bentzen SM, Agrawal RK, Aird EG, Barrett JM, Barrett-Lee PJ, Bentzen SM, et al.; START Trialists' Group. The UK Standardisation of Breast Radiotherapy (START) Trial B of radiotherapy hypofractionation for treatment of early breast cancer: a randomised trial. Lancet. 2008 Mar;371(9618):1098-107.

21 Common Terminology Criteria for Adverse Events v4.03 (CTCAE) [cited 2018 May 9]. Available from: https://ctep.cancer.gov/protocoldevelopment/electronic_applications/ ctc.htm\#ctc_archive.

22 Ferlay J, Soerjomataram I, Dikshit R, Eser S, Mathers C, Rebelo M, et al. Cancer incidence and mortality worldwide: sources, methods and major patterns in GLOBOCAN 2012. Int J Cancer. 2015 Mar;136(5):E359-86.

23 Barrios CH, Reinert T, Werutsky G. Global Breast Cancer Research: moving Forward. Am Soc Clin Oncol Educ Book. 2018 May; 38(38):441-50.

24 Piotrowski I, Kulcenty K, Wichtowski M, Murawa D, Suchorska W. Intraoperative Radiotherapy of Breast Cancer and Its Biological Effects. Breast Care (Basel). 2017 May;12(2): 109-13.

25 Pazos M, Schönecker S, Reitz D, Rogowski P, Niyazi M, Alongi F, et al. Recent Developments in Radiation Oncology: An Overview of Individualised Treatment Strategies in Breast Cancer. Breast Care (Basel). 2018 Aug; 13(4):285-91.

26 Akay BN, Sanli H, Heper AO. Postirradiation linear morphoea. Clin Exp Dermatol. 2010 Jun;35(4):35:106-8.

27 Spalek M, Jonska-Gmyrek J, Gałecki J. Radiation-induced morphea - a literature review. J Eur Acad Dermatol Venereol. 2015 Feb;29(2): 197-202.
28 Kushi J, Csuka ME. Generalized morphea after breast cancer radiation therapy. Case Rep Rheumatol. 2011;2011:951948.

29 Ardern-Jones MR, Black MM. Widespread morphoea following radiotherapy for carcinoma of the breast. Clin Exp Dermatol. 2003 Mar;28(2):160-2.

30 Bentzen SM, Skoczylas JZ, Overgaard M, Overgaard J. Radiotherapy-related lung fibrosis enhanced by tamoxifen. J Natl Cancer Inst. 1996 Jul;88(13):918-22.

31 Diago A, Llombart B, Requena C, Sanmartín $\mathrm{O}$, Guillén C. Postirradiation Morphea in $\mathrm{Pa}$ tients with Breast Cancer: Possible Association with Other Autoimmune Diseases. Actas Dermosifiliogr. 2019 Mar;110(2):153-9.

32 Schaffer JV, Carroll C, Dvoretsky I, Huether MJ, Girardi M. Postirradiation morphea of the breast presentation of two cases and review of the literature. Dermatology. 2000; 200(1):67-71

33 Riekki R, Jukkola A, Sassi ML, Höyhtyä M, Kallioinen M, Risteli J, et al. Modulation of skin collagen metabolism by irradiation: collagen synthesis is increased in irradiated human skin. Br J Dermatol. 2000 May;142(5): 874-80.

34 Fruchter R, Kurtzman DJ, Mazori DR, Wright NA, Patel M, Vleugels RA, et al. Characteristics and treatment of postirradiation morphea: A retrospective multicenter analysis. Am Acad Dermatol. 2017 Jan;76(1):19-21. 